\title{
EFFECT OF PRE-OPERATORY LOW-LEVEL LASER THERAPY ON PAIN, SWELLING, AND TRISMUS ASSOCIATED WITH THIRD-MOLAR SURGERY
}

\author{
Mohamed Samir Ali Hassan*, Ahmed Ahmed Hussein Al Feky**, Ahmed Mohamed Hosney ${ }^{* * *}$
}

\begin{abstract}
Objectives: The extraction of impacted third molars is commonly associated to pain, edema, trismus, limited jaw opening and movements. The objectives of this study is to verify if pre-surgical low-level laser therapy (LLLT) associated with the extraction of impacted lower third molars could add benefits to the postoperative symptoms. Subjects and Methods: Data from 24 patients subjected to a surgical extraction of lower third molars were pooled and divided into three groups. Patients that received only routine management were inserted in the control group. Group 1, patients received LLLT immediately after surgical removal of third molar and at 24 hours. In group 2 were included patients treated with LLLT immediately before the surgical removal of third molar and immediately after the end of the procedure. Results: Both laser-treated groups were characterized by minor events of post-surgery complications of pain, edema, trismus. Conclusions: Pre-surgical LLLT treatment seems to increase the analgesic effect of LLLT. However, trismus and edema were reduced in both laser treated groups, independently from the period of irradiation.
\end{abstract}

Key words: low-level laser therapy, mandibular third molar surgery, pain, swelling, trismus

\section{INTRODUCTION}

The extraction of impacted third molars is commonly associated to postsurgical transient complications of various intensity, like pain, edema, trismus, limited jaw opening and movements ${ }^{(1)}$. Traditionally these symptoms are treated by the use of glucocorticoids and NSAIDs, but their use should be limited especially in those patients with such disorders or those that already undergone extensive pharmacologic treatments ${ }^{(2)}$. Recent advancements in medicine have permitted the rapid development of light emitting devices for the control of pain and infections ${ }^{(3)}$; one of these is laser, in particular low-level laser therapy (LLLT) ${ }^{(4)}$. LLLT is able to modulate the inflammatory process without adverse effects, by reducing pain, swelling, and promoting the repair of damaged tissues ${ }^{(5)}$. Food and Drug Administration has approved the use of LLLT for pain relief in carpal tunnel syndrome since $2002^{(6)}$. Since then many data have been published and the use of LLLT for pain treatment developed in all medical fields ${ }^{(7-10)}$.

Studies have shown that postoperative LLLT with the use of a $980 \mathrm{~nm}$ diode laser was effective in the reduction of symptoms of pain, edema and trismus associated with third molars extraction ${ }^{(11-13)}$.

*B.D.S,2011G, Faculty of Dental Medicine, Al AzharUniversity “Assiut Dentist, Egyptian Ministry of Health, Egypt ** Assistant Professor of Oral and Maxillofacial Surgery Department, Faculty of Dental Medicine, Boys, Cairo Al-Azhar University

*** Lecturer of Oral and Maxillofacial Surgery Department, Faculty of Dental Medicine, Boys, Cairo Al-Azhar University 
However literature shows conflicting opinions about the effects of LLLT: a possible explanation is the fundamental importance of such parameters like the wavelength, the power density, the pulse structure and the irradiation time. Consequently, many results published in literature are negatives due to an incorrect choice of the light source or the best protocol. A systematic review and meta-analysis demonstrated that LLLT was effective in reducing pain, trismus, and swelling after mandibular third molar surgery. The heterogeneity of results in literature is probably a consequence of the various protocols and outcomes assessment and risk of bias of the different trials.

Moreover all studies in literature analyzed the effect of LLLT performed only after the third molars extraction, and actually no studies results about the effects of laser biostimulation also before the surgery. Many studies have highlighted the importance of preoperative anti-inflammatory treatments in reducing postoperative complications ${ }^{(14)}$. Preoperative low-intensity laser therapy reduced the number of postoperative inflammatory complications, hospital stay, severity of postoperative period in patients with benign prostatic hyperplasia ${ }^{(15)}$.

The aim of this study is to verify the better perioperatory protocol that is associated with minor complications, after the removal of impacted third molars.

\section{SUBJECTS AND METHODS}

In this study, data from 24 patients subjected to the surgical extraction of lower third molar over the period of one year from November 2018 to October 2019 in the Dental Clinics of Oral and Maxillofacial Surgery Department, Faculty of Dental Medicine, Boys, Cairo, Al Azhar University, were selected, pooled and analyzed. The protocol of this study was approved by the Faculty Ethical Committee. Criteria for exclusion included: systemic diseases, local and purulent infections, blood dyscrasia, previous or present gastric ulcers, heart disease, known hypersensitivities, allergies, or idiosyncratic reactions to any study medications, pregnancy and lactation. In addition, patients who had taken analgesics or anti-inflammatory drugs within 24 hours before surgery were not included in the study. The degree of surgical difficulty was assessed on pre-surgical orthopantomography using Pell-Gregory criteria: class III B and III C teeth, extractions that included open flap, odontotomy and osteotomy were included in the study ${ }^{(16)}$.

\section{Description of the standardized surgical procedure}

All patients before extraction had signed the informed consent and underwent routine preoperatory management that consisted in the disinfection of the surgical site with $10 \%$ povidoneiodine solution. Alveolar nerve block anesthesia was done by means of articaine hydrochloride + epinephrine $40 \mathrm{mg} / 0.01 \mathrm{mg} / \mathrm{ml}$, and infiltration of buccal soft tissues of the same local anesthetic (Laboratorios Inibsa, S.A. Spain).

Postoperatively, all patients were instructed to apply ice packs directly over the masseteric region on the operated side intermittently (10 minutes intervals). Chlorhexidine gluconate mouthwash was prescribed 3 times/day starting from the first day after the surgery until suture removal ( 7 days after the extraction). The antibiotic (Augmentin 1gm/12 hours for 5 days) treatment was prescribed.

Data from patients treated by the same surgeon were included in the study. Basing on pre- and postoperatory management, data were pooled and allocated into three groups for analysis and statistics.

Controls: patients treated only with routine management.

Group 1: patient subjected to laser irradiation within 10 minutes of completion of the extraction and at $24 \mathrm{~h}+$ routine management.

Group 2: patient subjected to the laser irradiation 10 minutes before the start of the surgery and also within 10 minutes of completion of the procedure 
with the same methodology + routine management. In all cases the operator who had performed the LLLT in all patients of group test was different from the surgeon; a third operator carried out all the measurements.

\section{LLLT protocol For treatment}

A diode laser device (model: LASOTRONIX, Model SMARTM. Poland) with a continuous wavelength of $980 \mathrm{~nm}$ and a therapy handpiece was used. Laser energy was applied at $300 \mathrm{~mW}(0.3 \mathrm{~W})$ for a total of $180 \mathrm{~s}, 60 \mathrm{~s}$ for each point, $0.3 \mathrm{~W} \times 180$ $\mathrm{s}=54 \mathrm{~J}$. Each LLLT session consisted of an intraoral and an extraoral phase ${ }^{(11)}$. Laser was applied $60 \mathrm{~s}$ extraorally at $1 \mathrm{~cm}$ from the skin over the area of the masseter, in the side that underwent surgery. Then laser was applied intraorally: $60 \mathrm{~s}$ on the lingual side of the alveolus of the teeth to be removed and other $60 \mathrm{~s}$ on the vestibular wall. The laser was used with circular movements and maintaining a constant distance of $1 \mathrm{~cm}$ from the gingiva.

\section{Evaluation of postoperative complications}

The pain referred by the patients at 24 hours and after 7 days was recorded through a visual analogue scale (VAS). Accordingly, pain was recorded as " 0 no pain" (patient experiences no discomfort) to "10extreme pain" (very noticeable pain which disturbs the patient's daily routine) Figure (1).

Swelling was evaluated for each patient and prior to surgery, with the aid of a measuring tape (in centimeters) by flexible meters product by IKEA as described by Gabka and Matsumara ${ }^{(28)}$. Three measurements were performed on the patient operated side using five reference points and calculating the average ${ }^{(29)}$.

The fixed points used were A; the most posterior point at the midline on the tragus, $\mathrm{B}$; lateral canthus of the eye, $\mathrm{C}$; the most lateral point on the corner of the mouth, D; soft tissue pogonium which is the most prominent point at the midline on the chin and $\mathrm{E}$; most inferior point on the angle of the mandible. The 3 lines were $\mathrm{AC}, \mathrm{AD}$ and $\mathrm{BE}$.
Distance between these points referred as (Line A, Line B, Line C) where: Line A, represents the distance between the lateral corner of the eye and the angle of the mandible(BE). Line B, represents the distance between the tragus and the outer corner of the mouth (the lip commissure) (AC). Line C, represents the distance between the tragus and the soft tissue pogonion (AD). The measurements were carried out just before the surgery and at postoperative days 1 and 7. Postoperative swelling was expressed as a percentage increase in facial width.

The trismus was evaluated measuring the interincisal opening, the maximal opening between the right maxillary and right mandibular central incisors before surgery. Postoperative trismus was measured as a percentage of the decrease in mouth opening.

Statistical analysis Statistical analysis was performed using SPSS for Windows (Statistical package for social science) version 23.0 (IBM SPSS Inc., Chicago, IL, USA). Analysis of variance (ANOVA) and the Bonferroni/ Dunn test were used to compare the parameters analyzed in the study. Data were analyzed using linear regression and descriptive statistics. The significance threshold was set at $\mathrm{p}=0.05$.

\section{RESULTS}

Lower scores in all studied parameters characterized both laser treated groups. Pain referred by patients using visual analog scale (VAS) as shown in figure (1). The mean pain score at 24 hours was significantly higher in controls (Median: 6.55 Range: 5.50-7.30) respect group 1) (Median: 5.00 Range: 4.00-6.50) and 2 (Median: 4.05 Range: 2.90-5.10) at 24 hours post-surgery. Study Groups showed significance decrease compared to Control group ( $\mathrm{p}=0.02,<0.001$ ) while Study Group (2) showed non significance compared to Study Group (1) $(\mathrm{p}=0.09)$. 


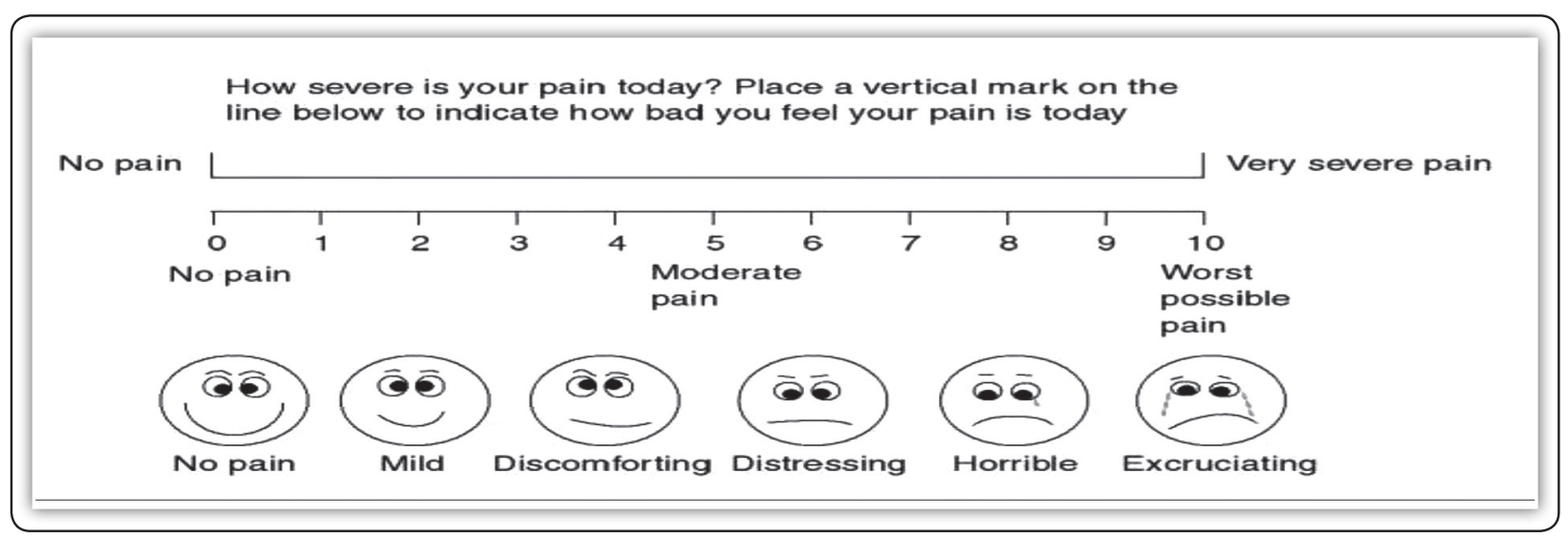

FIG (1) Shows the visual analog scale (VAS) of 10 point for pain measurement.

At $7^{\text {th }}$ postoperative day, no pain was found in the Study Group (1) \& Study Group (2) (Median: 1.70 Range: .60-2.50 ; Median: .55 Range: 1.101.60 respectively) and mild pain was detected in the control group (Median: 3.65 Range: 2.504.30). Study Group (1) \& Study Group (2) showed significant decrease compared to that in Control Group ( $\mathrm{P}=0.01,<0.001$ respectively) while Study Group (2) showed non significance compared to that in Study Group (1) ( $\mathrm{p}=0.07)$ as shown in figure (2).

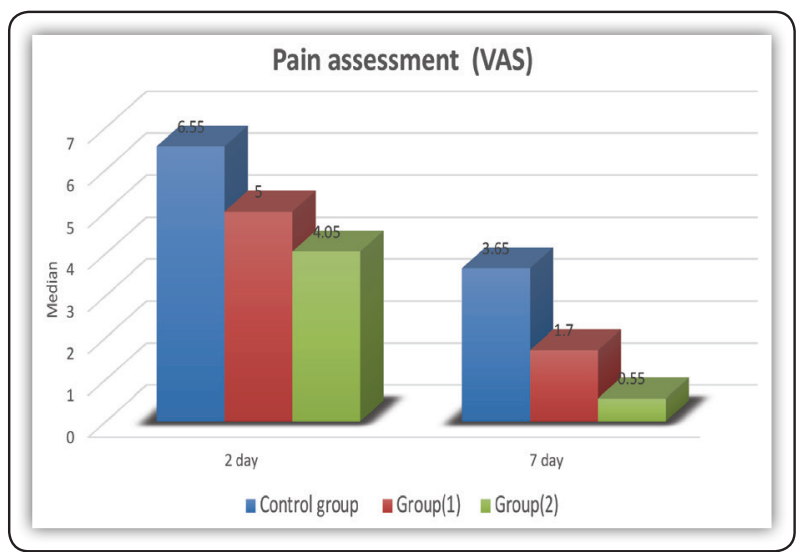

FIG (2) Comparison of VAS [2 days and 7 days] of Control group, Study Group(1) \& Study Group(2)

\section{Swelling:}

At $2^{\text {nd }}$ day, Sum of Lines of Study Group (1) \& Group (2) (Mean: 39.85 \pm SD: 0.48; Mean: 40.48 \pm SD: 0.41 respectively) showed significant decrease compared to that in Control Group (Mean: 44.15 \pm SD: 0.75$)(\mathrm{P}=<0.001,<0.001$ respectively $)$ while Study Group (2) (Mean: 40.48 \pm SD: 0.41) showed non significance compared to that in Study Group (1) (Mean: 39.85 \pm SD: 0.48) ( $\mathrm{p}=0.09)$.

At $7^{\text {th }}$ day, Sum of Lines of Study Group (1) \&

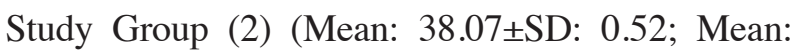
38.17 \pm SD: 0.43 respectively) showed significant decrease compared to that in Control Group (Mean: 42.26 \pm SD: 0.61$)(\mathrm{P}=<0.001,<0.001$ respectively $)$ while Study Group (2) (Mean: 38.17 \pm SD: 0.43) showed non significance compared to that in Study Group (1) (Mean: 38.07 \pm SD: 0.52) $(\mathrm{p}=0.91)$ as shown in figure (3).

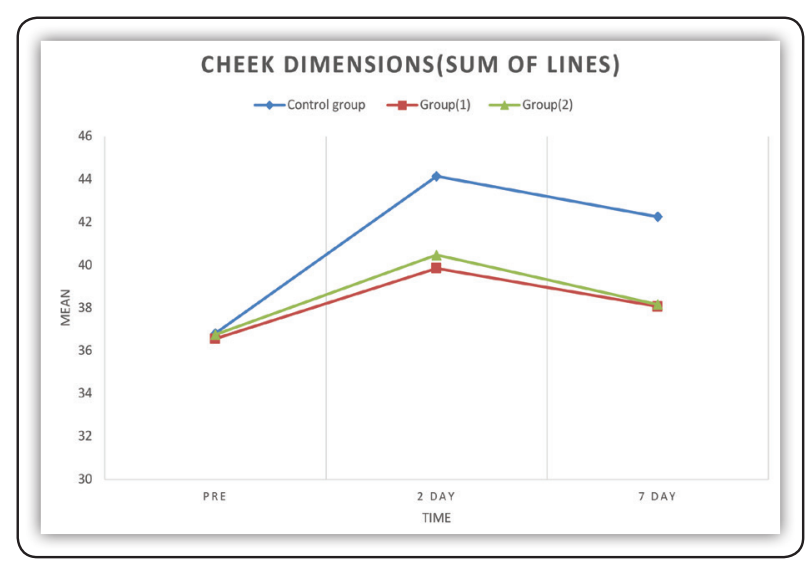

FIG (3) Comparison of Swelling between time intervals of Sum of lines within studied groups 


\section{Maximum Mouth Opening (MMO)}

Maximum Mouth Opening (MMO) was assessed preoperatively using caliper to measure the distance between the incisal edges of the upper and lower central incisors. Preoperative mean value was (47.22 \pm SD: 1.33$)$ in study Group (1) and (45.22 \pm SD: $2.59)$ in study Group (2) and (46.61 \pm SD: 2.39$)$ in the control group.

At the $2^{\text {nd }}$ postoperative day, the mean value of MMO of study Group (1) \& Group (2) (29.22 \pm SD: $3.00,25.09 \pm$ SD: 2.91 respectively) showed non significance when compared to Control group (Mean: $25.80 \pm$ SD: 2.37$),(\mathrm{p}=0.056,0.86$ respectively $)$ while Study Group (2) (Mean: 25.09 \pm SD: 2.91 respectively) showed significant decrease to Group (1) (Mean: 29.22 \pm SD: 3.00$)(\mathrm{p}=0.01)$. At the 7 th postoperative day, the mean value of mouth opening of study Group (1) ( Mean: 40.88 \pm SD: 1.67) showed significant increase when compared to Control group (Mean: 37.01 \pm SD: 2.55$)(\mathrm{p}=0.018)$ while study Group (2)

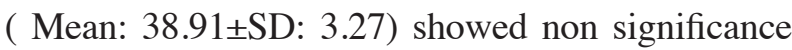

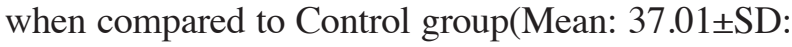
2.55) ( $\mathrm{p}=0.32$ ). Comparison of study Group (2) (Mean: $38.91 \pm$ SD: 3.27$)$ showed non significance when compared to study Group (1) group(Mean: 40.88 \pm SD: 1.67$)(\mathrm{p}=0.30)$.

\section{DISCUSSION}

A study, to verify the best peri-operatory protocol that was associated with minor complications, after the removal of impacted third molars, was conducted. We have found no studies in literature that analyzed the effects of pre-operatory LLLT on post-surgery complications after third molar removal. Our results confirmed that a double dose of LLLT, one immediately before and another after the surgery, is effective in reducing the perceived pain of patients and edema at $24 \mathrm{~h}$. There was no statistically significant differences between the group irradiated also in the pre-surgery phase (group 2) respect that irradiated only after the extraction (group 1), for the analyzed parameters.
Our results are in accordance with Koszowski ${ }^{(17)}$ that reported the comparison of analgesic effect of magnetic and laser stimulation before oral procedures. Laser stimulation and alternating magnetic field applied directly before oral surgery were-shown to be effective as analgesic agents to decrease intra- and postoperative sensations. Uspenskii $^{(18)}$ used low intensity laser light, (wavelength $675 \mathrm{~mm}$ ) as preoperative preparation in patients with surgical diseases of the lungs. He found many positive effects like antibacterial activity, reduction of endoscopic and morphological features of inflammation of the bronchial mucosa, stimulation of local immunity, the improvement of the respiratory system functional, positive dynamics of clinical status (a decrease of cough, dispnea, quantity of mucus discharge and haemoptysis). A possible explanation of analgesic effect of LLLT is the ability to modulate several signaling pathways and physiologic mechanisms involved in analgesia, like the increase of $\beta$-endorphin levels ( $\beta$-ep) and the modulation of biochemicals related to pain, including substance $\mathrm{P}(\mathrm{SP})$, tumor necrosis factor- $\alpha$ (TNF- $\alpha$ ), and cyclooxygenase-2 (COX-2) (19-21). Animal studies indicate that preoperative LLLT can act locally to prevent ischemic muscle damage by decreasing the activity of $\mathrm{CK}$ and the re-release of ROS, while increasing the levels of antioxidants and heat shock proteins ${ }^{(22)}$. For what concerning edema both irradiated groups were characterized by lower increase in facial width in the first 24 hours with statistically significant differences respect controls. The effect of LLLT in reducing postoperative edema is a direct consequence of the activation of lymphatic flow, and blood supply ${ }^{(23,24)}$. There were no additive effects in group 2 respect group 1 for what concerning facial edema. A possible explanation could be a latency time during which an additional laser irradiation is not able to induce additive effects on blood and lymphatic circulation. Indeed it has been shown that LLLT promotes the rapid increase in the number and diameter of the capillaries within the first hours until 
the peak at the twelfth hour after irradiation with low level lasers and the subsequent decrease to near-normal values ${ }^{(25)}$.

The percentage of decrease in mouth opening postsurgery seem to be relatively influenced by LLLT. Both group 1 and 2 are characterized by a lower percentage at 24 hours respect controls, but no statistically significant differences have been found. The same results was recorded at 7 days and the preoperative irradiation in adjunction to the postoperative one seems to permit a faster recovery of the interincisal opening. The reduction of this parameter is a direct consequence of trismus that derive from inflammation after surgical procedures but it is also caused by the persistent permanence of the patient with opened mouth which fatigues the elevator muscles of the jaw and temporomandibular joint (TMJ). LLLT is reported to induce muscle relaxation ${ }^{(26)}$. In particular, some researchers have reported that applying the laser on muscles before the fatigue-inducing exercise provides more satisfactory reduction of fatigue ${ }^{(27)}$. However, in this study only masseter and medial pterygoid muscle were irradiated. For this reason in order to have greater effects on interincisal TMJ opening other elevators muscles should be irradiated.

\section{CONCLUSION}

1. Results confirm the usefulness of low-level laser therapy in reducing post-surgery complications of pain, edema and trismus.

2. A preoperative irradiation immediately before and another after the extraction seems to increase the analgesic effect of LLLT. Trismus and edema are reduced in both laser treated groups. However, the preoperative LLLT seems not to confer additional benefits respect postoperatory irradiation.

3. Results suggest that LLLT could represent a viable tool for the control of pain in those patients for which pharmacological treatment is be contraindicated.

\section{REFERENCES}

1. Piecuch JF. What strategies are helpful in the operative management of third molars? J Oral Maxillofac Surg. 2012;70:S25-32.

2. Kim K, Brar P, Jakubowski J, Kaltman S, Lopez E. The use of corticosteroids and nonsteroidal antiinflammatory medication for the management of pain and inflammation after third molar surgery: a review of the literature. Oral Surg Oral Med Oral Pathol Oral Radiol Endod. 2009;107:630-40.

3. D'Ercole S, Spoto G, Trentini P, Tripodi D, Petrini M. In vitro inactivation of Enterococcus faecalis with a LED device. J Photochem Photobiol B. 2016;160:172-7. Med Oral Patol Oral Cir Bucal. 2017 Jul 1;22 (4):e467-72. The analgesic effect of low level laser therapy e472

4. Chow R, Armati P, Laakso EL, Bjordal JM, Baxter GD. Inhibitory effects of laser irradiation on peripheral mammalian nerves and relevance to analgesic effects: a systematic review. Photomed Laser Surg. 2011; 29:365-81.

5. Oliveira Sierra S, Melo Deana A, Mesquita Ferrari RA, Maia Albarello P, Bussadori SK, Santos Fernandes KP. Effect of low-level laser therapy on the post-surgical inflammatory process after third molar removal: study protocol for a double-blind randomized controlled trial. Trials. 2013; 14:373.

6. Asnaashari M, Safavi N. Application of Low level Lasers in Dentistry (Endodontic). J Lasers Med Sci.2013;4:57-66

7. Elbay ÜŞ, Tak Ö, Elbay M, Uğurluel C, Kaya C. Efficacy of LowLevel Laser Therapy in the Management of Postoperative Pain in Children After Primary Teeth Extraction: A Randomized Clinical Trial. Photomed Laser Surg. 2016;34:171-7.

8. Cotler HB, Chow RT, Hamblin MR, Carroll J. The Use of Low Level Laser Therapy (LLLT) For Musculoskeletal Pain. MOJ Orthop Rheumatol. 2015;5:00068.

9. Baltzer AW, Ostapczuk MS, Stosch D. Positive effects of low level laser therapy (LLLT) on Bouchard's and Heberden's osteoarthritis. Lasers Surg Med. 2016;48:498

10. Stasinopoulos D, Papadopoulos K, Lamnisos D, Stergioulas A. LLLT for the management of patients with ankylosing spondylitis. Lasers Med Sci. 2016;31:459-69.

11. Ferrante M, Petrini M, Trentini P, Perfetti G, Spoto G. Effect of low-level laser therapy after extraction of impacted lower third molars. Lasers Med Sci. 2013;28:845.

12. Avci P, Gupta A, Sadasivam M, Vecchio D, Pam Z, Pam $\mathrm{N}$, et al. Low-level laser (light) therapy (LLLT) in skin: stimulating, healing, restoring. Semin Cutan Med Surg. 2013;32:41-52. 
13. He WL, Yu FY, Li CJ, Pan J, Zhuang R, Duan PJ. A systematic review and meta-analysis on the efficacy of lowlevel laser therapy in the management of complication after mandibular third molar surgery. Lasers Med Sci. 2015;30:1779-88.

14. Rana MV, Desai R, Tran L, Davis D. Perioperative Pain Control in the Ambulatory Setting. Curr Pain Headache Rep. 2016;20:18.

15. Neŭmark AI, Muzalevskaia NI. [Low-intensity laser radiation in preoperative preparation of patients with benign prostatic hyperplasia]. Urologiia. 2000;1:11-5.

16. Almendros-Marqués N, Berini-Aytés L, Gay-Escoda C. Influence of lower third molar position on the incidence of preoperative complications. Oral Surg Oral Med Oral Pathol Oral Radiol Endod. 2006;102:725-32.

17. Koszowski R, Smieszek-Wilczewska J, Dawiec G. Comparison of analgesic effect of magnetic and laser stimulation before oral surgery procedures. Wiad Lek. 2006;59:630-3.

18. Uspenskiǔ LV, Chistov LV, Kogan EA, Loshchenov VB, Ablitsov IuA, Rybin VK, et al. Endobronchial laser therapy in complex preoperative preparation of patients with lung diseases. Khirurgiia (Mosk). 2000;2:38-40.

19. Camillo de Carvalho PT, Leal-Junior ECP, Alves ACA, De Melo Rambo CS, Sampaio LMM, Oliveira CS, et al. Effect of low-level laser therapy on pain, quality of life and sleep in patients with fibromyalgia: study protocol for a double-blinded randomized controlled trial.Trials. 2012;13:221.

20. Gür A, Karakoç M, Nas K, Cevik R, Saraç J, Demir E. Efficacy of low power laser therapy in fibromyalgia: a single-blind, placebocontrolled trial. Lasers Med Sci. 2002;17:57-61.
21. Hsieh YL, Hong CZ, Chou LW, Yang SA, Yang CC. Fluence-dependent effects of low-level laser therapy in myofascial trigger spots on modulation of biochemical associated with pain in a rabbit model. Lasers Med Sci. 2015;30:209-16.

22. Rizzi CF, Mauriz JL, Freitas Corre^a DS, Moreira AJ, Zettler CG, Filippin LI, et al. Effects of low-level laser therapy (LLLT) on the nuclear (NF)- kappaB signaling pathway in traumatized muscle. Lasers Surg. Med. 2006;38:704-13.

23. Tomaz de Magalhães M, Núñez SC, Kato IT, Ribeiro MS. Light therapy modulates serotonin levels and blood flow in women with headache. A preliminary study. Exp Biol Med (Maywood) 2016;241:40-5.

24. Smoot B, Chiavola-Larson L, Lee J, Manibusan H, Allen DD. Effect of low-level laser therapy on pain and swelling in women with breast cancer-related lymphedema: a systematic review and metaanalysis. J Cancer Surviv.2015;9:287-304.

25. Ihsan FR. Low-level laser therapy accelerates collateral circulation and enhances microcirculation. Photomed Laser Surg. 2005;23:289-94.

26. Herpich CM, Leal-Junior ECP, Amaral AP, de Paiva TJ, dos Santos Glória IP, Garcia MBS, et al. Effects of phototherapy on muscle activity and pain in individuals with temporo-mandibular disorder: a study protocol for a randomized controlled trial. Trials. 2014;15:491.

27. Leal Junior EC, Lopes-Martins RA, Frigo L, De Marchi T, Rossi RP, de Godoi V, et al. Effects of low-level laser therapy (LLLT) in the development of exercise-induced skeletal muscle fatigue and changes in biochemical markers related to post-exercise recovery. J Orthop Sports Phys. Ther. 2010;40:524-32. 